\title{
Infrared image processing devoted to thermal non-contact characterization- A connecting thread for the I2M-Bordeaux activities in the field of IR thermography
}

\author{
by JC. Batsale*, C. Pradere** \\ * Arts et Métiers Paris Tech, I2M, UMR 5295, F-33400 Talence, France, jean-christophe.batsale@ensam.eu \\ ${ }^{\star *}$ CNRS, I2M, UMR 5295, F-33400 Talence, France, Christophe.pradere@ensam.eu
}

\begin{abstract}
Generally the IR images are related to the transient temperature field related to the emission of a black surface in response to an external or internal heating (active IR thermography). At I2M-Bordeaux, the first applications were devoted to the so called thermal Non-Destructive Evaluation methods. But with simplified assumptions related to the transverse diffusion, the in-plane diffusion and transport phenomena can be also considered and applied to microfluidics or multispectral detection. There are a lot of possible strategies to process the big amount of temperature data (previous integral transformation of the images, compression, elimination of the non useful areas...), generally based on the necessity to analyse the derivative of the temperature field versus space and time.
\end{abstract}

\section{Introduction}

A 2D temperature field can be the signature of a lot of heat transfer phenomena at the surface of a solid or a liquid (heat conduction or diffusion through homogeneous or heterogeneous media, convective transport in complex systems...). One of the main intuitive way to process the signal is to study the time or space derivatives of such fields, in order to link the temperature observation to a heat transfer model. Several strategies for the temperature field processing in space and in time will be illustrated on a suitable analytical temperature field. The application examples devoted to Non-Destructive Evaluation, microfluidic systems and multispectral tomography subsequently presented are based on a derivation of the experimental signal. Such examples are also illustrating the main domains approached in our team in Bordeaux University (I2M institute).

\section{Thermal processing of a $2 \mathrm{D}$ transient $T(x, y, t)$ field}

From a $2 \mathrm{D}$ transient $T(x, y, t)$ field, and a heat transfer model (even simplified) is it often possible to estimate a resultant thermophysical properties field. The diffusion and convection transport modes can be considered in order to set out an identification model. The processing of such fields needs of course to be aware of the noise and perturbations considerations about the temperature field recording. The random noise with a zero mean and a uniform and diagonal covariance matrix will generally be taken into account. Several aspects related to the "bad pixels", the space or time correlation and the non-regular time steps will here be assumed to be previously circumvented. Maybe, the first "natural" processing of such data is to try to set out the derivative (versus space or time) of such a discrete temperature field. The space or time derivation of noisy fields is a difficult task, because such operator is amplifying the random measurement noise, if no precaution is taken. A "filtering" is then necessary, but the risk is to lose a part of the original information. Several strategies will be here examined (the finite differences, the polynomial fit, the orthogonal basis decomposition and the convolution), in order to numerically implement the derivation of a discrete signal.

In order to test and illustrate the processing possibilities, it is here proposed to consider the 1D temperature relaxation of a solution to a system such as:

$$
a \frac{\partial^{2} T}{\partial x^{2}}=\frac{\partial T}{\partial t}
$$

with: $T(x, t=0)=1$ if $0<x<b \quad$ and $T(x, t=0)=0$ if $b<x<L$; and at $x=0$ and $x=L:\left.\quad \frac{\partial T}{\partial x}\right|_{0}=0$ and $\left.\frac{\partial T}{\partial x}\right|_{L}=0$

After a time $t$, the temperature field is relaxed (or filtered) by diffusion, and an approximation of the field is then:

with:

$$
T(x, t)=b / L+2 / L \sum_{n=1}^{N} \frac{\sin \left(\alpha_{n} b\right)}{\alpha_{n}} \exp \left(-a \alpha_{n}^{2} t\right) \cos \left(\alpha_{n} x\right)
$$

$$
\alpha_{n}=n \pi / L \text { and } n, N \text { finite integers. }
$$


Rigorously, the previous expression is a serie with $N$ tending to infinity. A physical filter (due to the exponential term) is acting here and limiting the space-frequency content of the signal. The parameter which allows to control the filter is here the observation time. This observation time will be fixed in this section and only the space field will be considered.

The observed temperature is a vector obtained from the previous expression at regularly spaced space steps $\Delta x$ and a white gaussian noise is added.

A-

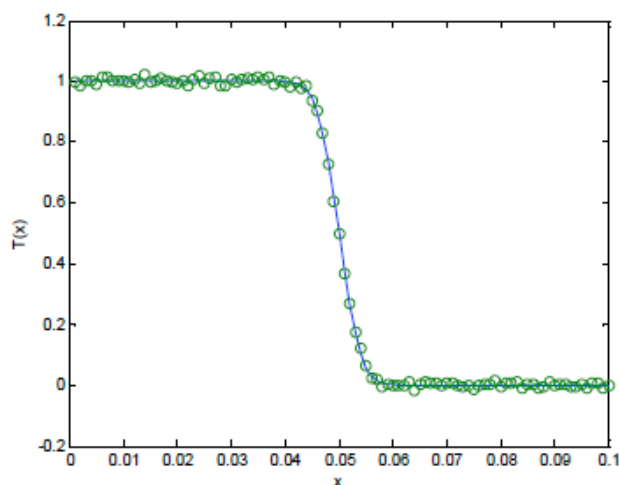

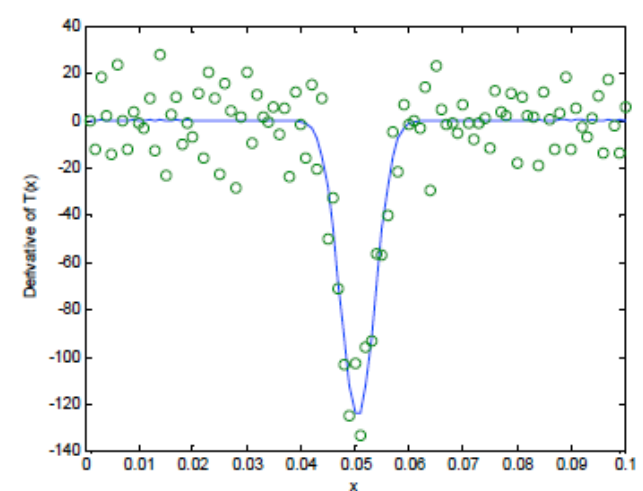

expression at time $t=0.5 \mathrm{~s} ; a=10^{-5} \mathrm{~m}^{2} \mathrm{~s}^{-1} ; b=L / 2 ; L=0.1 \mathrm{~m}$; (continuous line: real signal, 'o': discrete noisy signal); $B$-Derivative of the previous noisy signal by finite differences.

The initial gaussian noise is with zero mean and $s=0.01$. The resulting noise is amplified by the derivation operation.

\subsection{Finite differences}

The finite difference approach used on figure 1 is generally presented at each space step $i$, as:

$$
\hat{T}_{i}^{\prime}=\frac{\hat{T}_{i+1}-\hat{T}_{i}}{\Delta x}
$$

If it is assumed, that the relation between the observed temperature $\hat{T}_{i}$ and the real temperature $T_{i}$ is:

$$
\hat{T}_{i}=T_{i}+e_{T_{i}}
$$

with $e_{T_{i}}$ representing the random variable related to the gaussian noise, uniform whatever the position $x_{i}$. The asymptotic expansion around $x_{i}$ is such as:

$$
\hat{T}_{i}^{\prime}=\frac{T\left(x_{i+1}\right)-T\left(x_{i}\right)}{\Delta x}+\varepsilon\left(x_{i+1}\right)+\frac{e_{T i+1}-e_{T i}}{\Delta x} \quad \text { With: } \quad \lim _{x \rightarrow x_{i}} \varepsilon(x)=0
$$

Two kinds of errors have then to be considered: the approximation error $\varepsilon\left(x_{i}\right)$ related to the rest of the asymptotic expansion and the random error related to the random variable $e_{T_{i}}$. Unfortunately, when the space step $\Delta x$ is tending to zero, the approximation error is effectively tending to zero, but the random error is tending to infinity!

The difference of two random variables is a linear operation which amplify the initial noise. In order to avoid such a difficulty, it is necessary to "filter" the signal or to project the discrete observed information in a basis of functions. One of the simplest basis of function can be a polynomial basis.

\subsection{Polynomial fitting}

From the same previous measurements, a polynomial fitting can be implemented such as:

$$
T(x)=\sum_{n=0}^{N} \beta_{n} x^{n}
$$

The estimation of the $\beta_{n}$ parameters in vector: $\mathbf{B}=\left[\beta_{1}, \beta_{2}, \beta_{3} \ldots . \beta_{n}\right]^{T}$ can then be obtained by a linear least-square relation such as :

$$
B=\left(X^{t} \mathbf{X}\right)^{-1} \mathbf{X}^{\mathrm{t}} \hat{\mathbf{T}}
$$

With: 
In order to minimize the distance:

$$
\mathbf{X}=\left[\begin{array}{cccc}
1 & x_{1} & x_{1}{ }^{2} & \cdots \\
1 & x_{2} & x_{2}{ }^{2} & \cdots \\
\cdot & & & \\
1 & x_{m} & x_{m}{ }^{2} & \cdots
\end{array}\right]
$$

$$
\|\hat{\mathbf{T}}-\mathbf{X B}\|=(\hat{\mathbf{T}}-\mathbf{X B})^{T}(\hat{\mathbf{T}}-\mathbf{X B})
$$

The construction of the derivative will then consist in considering the derivative of the polynomial function. The Matlab software is convenient in order to implement such calculations because the $\mathbf{X}^{\mathbf{t}} \mathbf{X}$ matrix can be bad conditioned (Wandermonde matrix) and must be inversed with special precautions. One example of such processing is shown on the figure 2.

A-
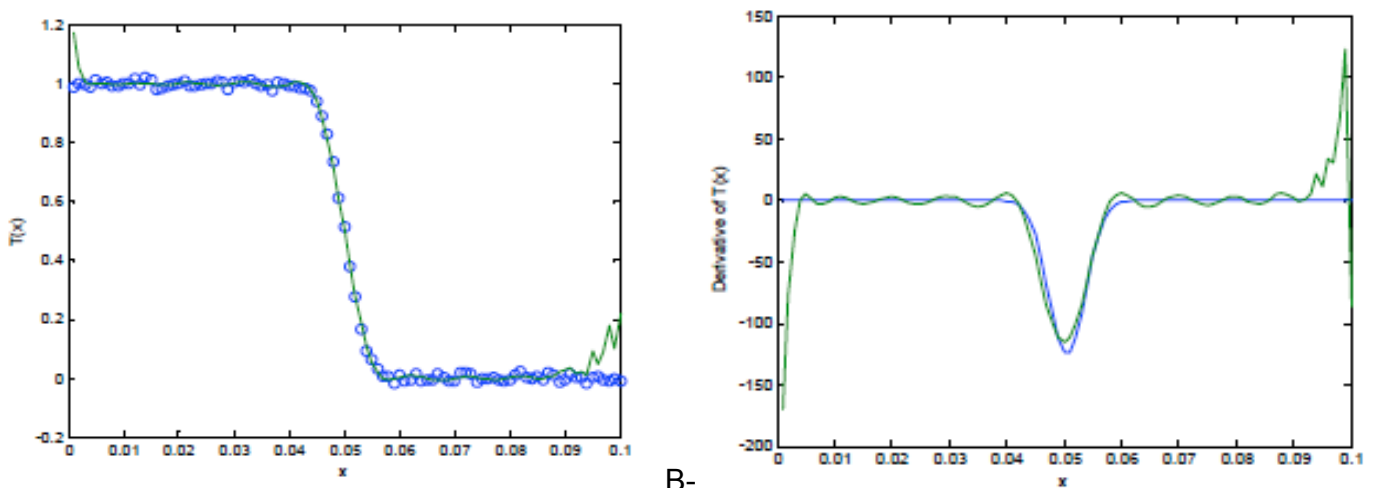

B$N=30$. The number of observation points is 100

It is shown here that the direct derivation of the estimated polynomial expression is giving a suitable continuous approximation in the considered domain. Approximation errors are occurring at the boundary of the domain if no precautions or assumptions are taken. One other strategy is to chose a basis "near from the considered physical phenomenon". Here, the ideal basis is the basis made of the Fourier cosine functions, because the cosine vectors are here verifying the boundary conditions (null derivative at $x=0$ and $x=L$ ) and are also the eigenvectors of the diffusion phenomenon (eigenvectors of the Laplacian operator in Cartesian coordinates and adiabatic boundaries).

\subsection{Fourier cosine basis}

The same processing as the polynomial fitting can be considered with such an expression:

$$
T(x)=\sum_{n=0}^{M} \beta_{n} \cos \left(\alpha_{n} x\right) \text { with: } \alpha_{n}=n \pi / L
$$

The estimation of the parameter vector: $\mathbf{B}=\left[\beta_{1}, \beta_{2}, \beta_{3} \ldots . \beta_{M}\right]^{T}$ is then obtained by the same expressions as previously (in section 2.2), excepted that the $\mathbf{X}^{t} \mathbf{X}$ matrix is orthogonal, and then very easy to be inverted. The covariance matrix related to the parameters is then as diagonal as the observable vector. The result of the estimation is then, with 30 terms for the serie, illustrated on figure 3 .

A-

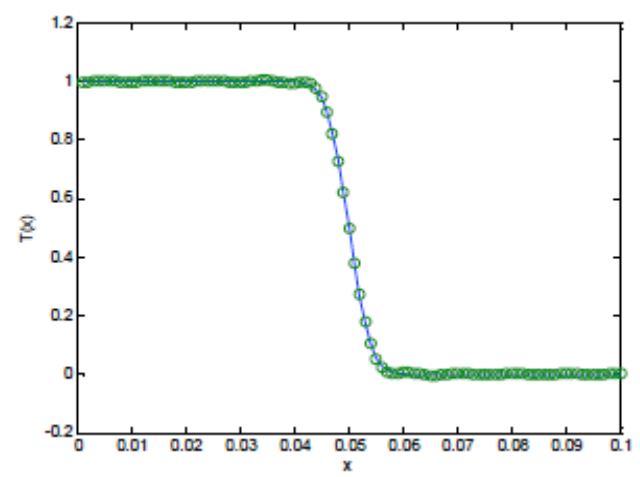
B-

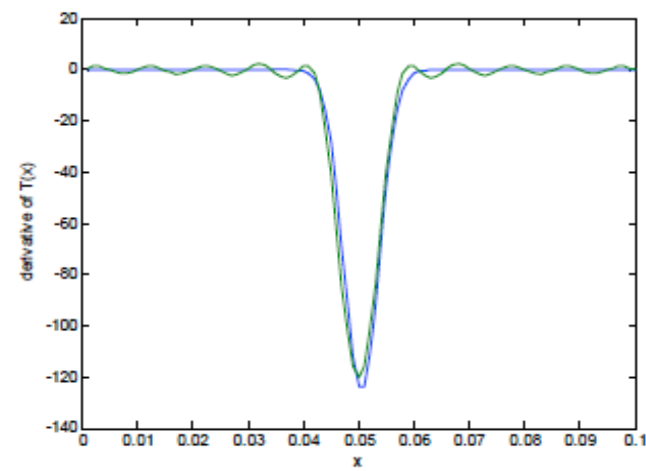
observation points is 100 . 


\subsection{Filtering with a convolution kernel}

The filtering is a usual operation in signal processing, which consist in weighting the signal with a moving average. It must be noticed that the observable signal himself is maybe previously filtered by the proper instrument. A new approximation of the signal can then be considered by $\widetilde{T}(x)$ such as :

$$
\widetilde{T}(x)=\int_{0}^{L} p(\chi) T(x-\chi) d \chi
$$

$p(x)$ must be normed such as :

$$
\int_{0}^{L} p(\chi) d \chi=1
$$

The derivative of $\widetilde{T}(x)$ is then conveniently considered by the commutability of the convolution product such as :

$$
\frac{d \tilde{T}}{d x}(x)=\int_{0}^{L} p(\chi) \frac{d T(x-\chi)}{d x} d \chi=\int_{0}^{L} T(\chi) \frac{d p(x-\chi)}{d x} d \chi
$$

The discrete approximation of the derivative is then conveniently considered by a convolution with a " derived » kernel. One very simple illustration is given on figure 4 . The discrete convolution kernel of the filter is for example [ $\left.\begin{array}{lllll}1 & 2 & 1\end{array}\right] / 4$ and an approximation of the convolution kernel for the derivation is then [1/2 $0-1 / 2]$. It can be noticed that this "slight" convolution (affecting only a few number of neighbours and very similar to the finite difference method) allows to obtain good results with less effort. The main problem is to find a compromise between the bias caused by the filtering and the perturbation caused by the noise.

A-
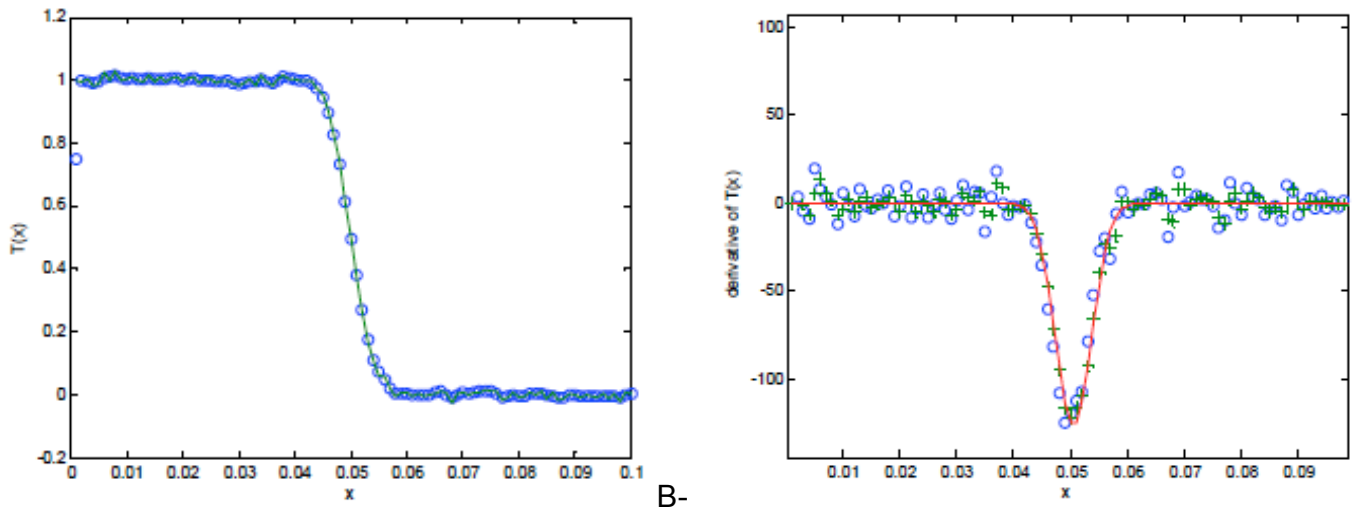

A-

B-

[1/2 0 -1/2] (plot'o') and comparison with the finite difference derivative of the previously filtered signal (plot'+').

\subsection{Singular Value Decomposition (SVD) of the whole space and time signal}

The previous methods consists in finding a compromise between the "approximation error" and the "filtering". The number of terms of the serie (or the rank of basis), or the width of the convolution kernel are biasing the signal if they are "used too far". A lot of other methods can be considered (for example more sophisticated regularisation techniques, see [1]). When a large field must be processed the choice of the compromise between the filtering and the bias is made by trial and error. The key point is the knowledge of the random noise (at the minimum the standard deviation). Often, the experiencer does not know the characteristic of the noise in his proper experience. It is then difficult to implement an optimal filtering of the signal. One way to separate the "available signal" from the "random noise", when the experiencer has a great amount of space and time information, consists in implementing the singular value decomposition (SVD) of the $T(x, t)$ field, or the $\hat{T}\left(x_{i}, t_{j}\right)$ discrete observable matrix.

Applying the SVD to temperatures matrix $\hat{\mathbf{T}}$ yields:

$$
\hat{\mathbf{T}}_{n, m}=U_{n X n} \Sigma_{n \times n} V_{n, m}^{T}
$$

Where $\Sigma_{n \times n}$ is a diagonal matrix 
The modes $U_{k}$ and $V_{i}$ are the column vectors of matrices $\boldsymbol{U}$ and $\boldsymbol{V}$ respectively, and the singular values $\gamma_{k}$ are the diagonal elements of $\Sigma_{n \times n}$ arranged in descending order.

Such a decomposition of the global space/time field is offering a lot of advantages. First, the examination of the singular values $\gamma_{k}$ allows to select the really available signal. Thermal phenomena are often related to diffusion problems (naturally filtered), with only a few available singular values. The decomposition obtained with the reduced number of singular values is then offering a reduced representation of the field which allows a lot of possibilities (reduced computational effort for the further parameters estimations, low memory storage, new orthogonal basis and projection possibilities, see [2] to [5] ). In the case illustrated on figure 5, the SVD is allowing an optimal filtering without any previous knowledge about the random noise at any time. The finite differences derivative is then available without precautions.

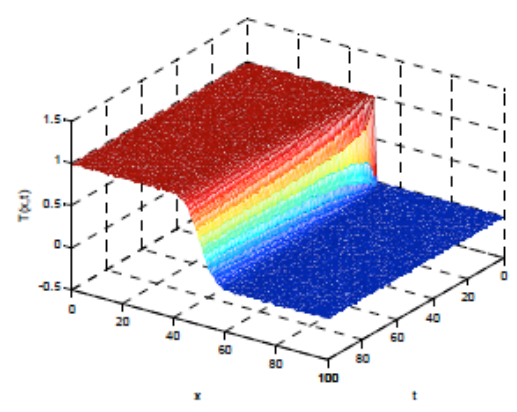

A-

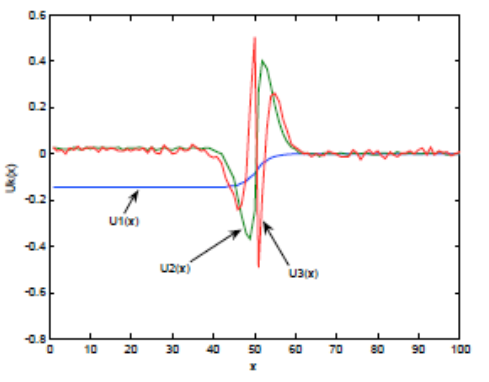

\section{C-}

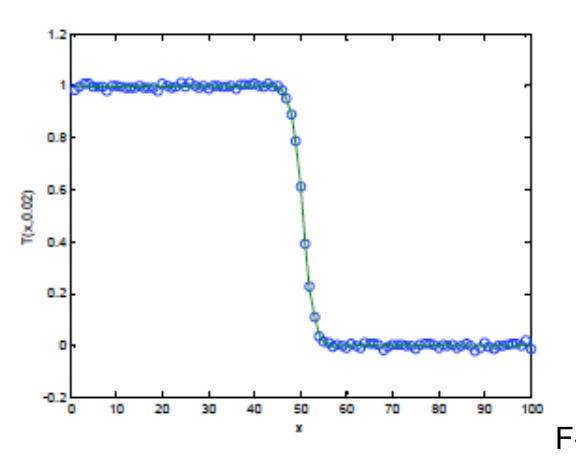

E-

B-
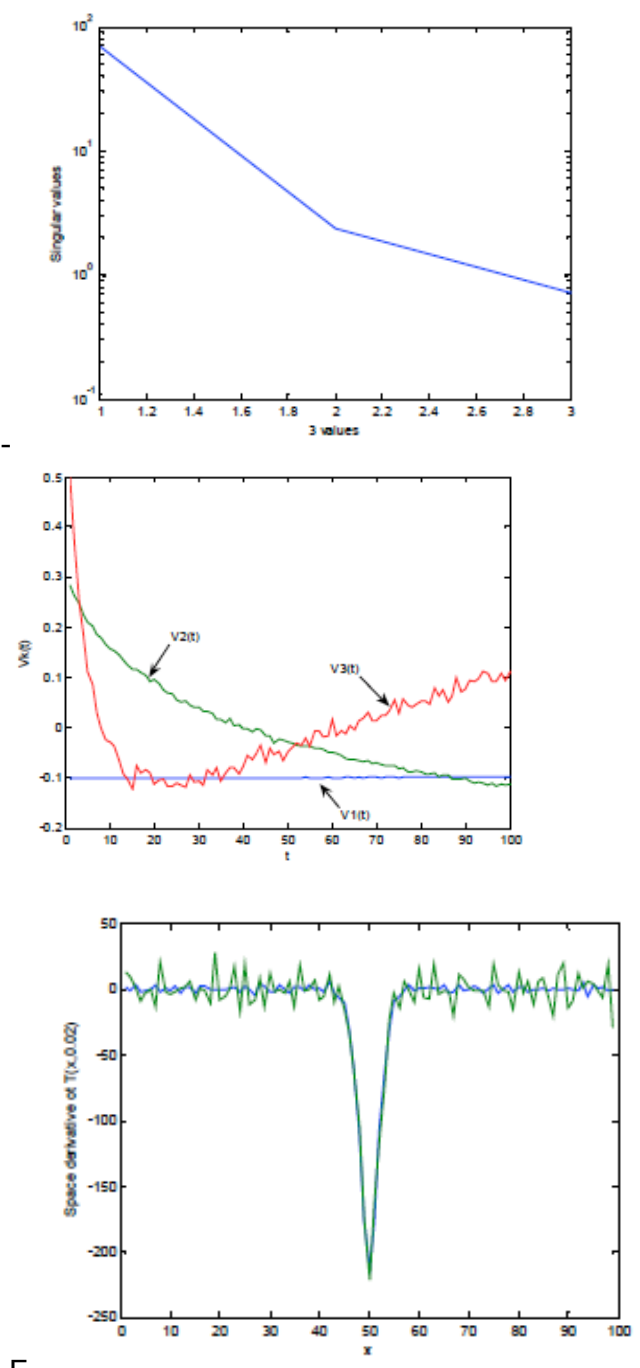

Fig 5: A- $T(x, t)$ noisy temperature field (time extention from figure 5), B-3 first singular values,C-D-First $\boldsymbol{U}$ and $\boldsymbol{V}$ vectors, coming from the SVD decomposition, E- Reconstruction of the previous signal at time $t=0.02 s$, with 3 term of the SVD decomposition.F"Noisy" derivation of the previous signal (initial noisy observation) compared the finite differences derivative of the SVD reconstruction)

The previous example is showing that the transient situation even non-stationnary, is giving a greater amount of data than a unique image. The SVD decomposition is a mean to squize and to process the great amount of data quite with the same effort as in the stationary case. It must be noticed that the orthogonal basis $\boldsymbol{U}$ is more reduced than the basis of cosine functions used in section 2-4.

\section{Applications}


The approaches previously cited (finite differences, polynomial decomposition, Fourier analysis, Singular Value Decomposition, convolution ...) are not only a range of tools for the derivation of the temperature fields but also widely used in classical applications for the processing of the fields recorded with infrared cameras. In the case of Nondestructive evaluation with the 1D flash experiment, the time-logarithmic derivative of the transient signal is related to a transverse diffusivity variation which gives a suitable decomposition of the signal. Very often, the "temperature contrast" related to a defect detection can be considered with a derivation operator. In the case of microfluidic devices characterization, it is necessary to discriminate and calibrate the convective transport in micro-channels from the purely diffusive in-plane heat transport. The comparison between a first derivative (convective transport) and a second derivative (diffusive transport) must be considered. More recently, the transmission of wide-band multispectral infrared sources through heterogeneous objects can be detected by simple thin opaque screens where the temperature field must be processed in order to avoid the in plane diffusion. Once again the time and space derivatives of the temperature field will be considered.

\subsection{Application to NDE}

Non Destructive Evaluation (NDE) with infrared cameras consists generally to apply a heat pulse on a non homogeneous parallelepipedic slab and to process the temperature response $T(x, y, t)$ from one face of the sample (the front face or the rear face) (see [6]). The aim of such processing is to estimate some characteristics of the heterogeneities (structure, size, nature, position inside the sample...). Instead of a 3D heterogeneous geometry, it is often convenient to consider thin samples with thin heterogeneities such as the transfer is locally 1D (versus $z$ direction). One asymptotic expansion assuming that the heterogeneities fluctuations are small compared to the mean value of one thermophysical property of the sample yields a linear relationship, such as:

$$
T(x, y, z, t)=q(x, y)\left(f\left(z, t, \beta_{0}\right)+\left.\Delta \beta(x, y) \frac{\partial f}{\partial \beta}\right|_{\left(z, t, \beta_{0}\right)}\right)
$$

Where the functions $q(x, y)$ is the spatial distribution of energy coming from a flash excitation and $\Delta \beta(x, y)$ is the spatial thermophysical property variation(diffusivity, conductivity, thickness...). $q(x, y)$ and $\Delta \beta(x, y)$ have to be specified with a finite number of parameters which will be the object of the estimation procedure. Such procedure will then consists in processing the weighted sum of the images $T(x, y, z, t)$ where the weighting functions are the sensitivity functions $f\left(z, t, \beta_{0}\right)$ and $\partial f /\left.\partial \beta\right|_{\left(z, t, \beta_{0}\right)}$. The temperature response of the front or the rear face of the sample will be recorded by a camera in order to estimate a map or a field of thermophysical properties. In the ideal case, the sample (a plane plate of small thickness $L$ ) is assumed to be thermally insulated and with a temperature field initially uniform $(T(x, y, z, t=0)=0)$. If the heat transfer is supposed 1-D, then, the temperature response related to a unique location $(x, y)$ corresponding to a pixel, to an instantaneous thermal pulse, is given (See [7]) on the front face $(z=0)$ :

$$
T(z=0, t)=\frac{Q}{\rho c L} \cdot\left(1+2 \cdot \sum_{n=1}^{\infty} \exp \left(-\frac{n \cdot \pi \cdot a \cdot t}{L^{2}}\right)\right)=\frac{Q}{\rho c L} f\left(a t / L^{2}\right)
$$

From the previous expression, the estimation problem of several parameters can be considered. Instead of the thermal diffusivity, the estimation problem of the sample thickness $L$, the thermal conductivity $\lambda$ and the volumic heat capacity $\rho \mathrm{c}$ can be considered. In each case, a reference approximated value of the parameter must be known (and noted: $L_{0}$, $\lambda_{0}$ and $\rho \mathrm{c}_{0}$ ). In many cases such reference values can be obtained by a previous global measurement. The following first order asymptotic expansions can be for example written for the front face, at each time $t_{i}$ : For example, if a thermal conductivity variation is to be estimated (see[8]):

$$
T\left(0, t_{i}\right) \approx \frac{Q}{\rho c L} f\left(\lambda_{0} t_{i} / \rho c L^{2}\right)+\left.\frac{Q}{\rho c L} \frac{\Delta \lambda}{\lambda_{0}} t \frac{\partial f}{\partial t}\right|_{\left(\lambda_{0} t_{i} / \rho c L^{2}\right)}
$$

It is very important to notice that it is possible to replace the sensitivity functions $f\left(z=0, t, \beta_{0}\right)$ and $\partial f /\left.\partial \beta\right|_{\left(z, t, \beta_{0}\right)}$ by a linear combination of $f\left(z=0, t, \beta_{0}\right)$ and the time logarithmic derivative $t . \partial f /\left.\partial t\right|_{\left(z=0, t, \beta_{0}\right)}$. It is then possible to implement a linear relationship such as:

$$
T\left(0, t_{i}\right) \approx \beta_{1} X_{\beta_{1}}\left(t_{i}\right)+\beta_{2} X_{\beta_{2}}\left(t_{i}\right)
$$

The functions $X_{\beta_{j}}\left(t_{i}\right)$ are the sensitivity functions of $T\left(0, t_{i}\right)$ to parameters $\beta_{j}$. If other parameters combination estimation (such as thermal diffusivity estimation) is considered, the resulting sensitivity function will be a linear combination of functions $f$ and $t(\partial f / \partial t)$.

The parameters $\beta_{j}$ are defined in each estimation situation such as:

$$
\beta_{1}=\frac{Q}{\rho c L} \text { and } \beta_{2}=\frac{Q}{\rho c L} \frac{\Delta \lambda}{\lambda_{0}}
$$

That is to say with matrix notation, considering the vectors and matrices: 


$$
\mathbf{T}=\left[\begin{array}{lll}
T\left(0, t_{1}\right) & \ldots & T\left(0, t_{N}\right)
\end{array}\right]^{t}, \mathbf{X}=\left[\begin{array}{ccc}
X_{\beta_{1}}\left(t_{1}\right) & \ldots & X_{\beta_{1}}\left(t_{N}\right) \\
X_{\beta_{2}}\left(t_{1}\right) & \ldots & X_{\beta_{2}}\left(t_{N}\right)
\end{array}\right]^{t},
$$

It yields under matrix notations:

$$
\mathbf{T}=\mathbf{X}\left[\begin{array}{l}
\beta_{1} \\
\beta_{2}
\end{array}\right]
$$

If the measurement noise on each component of the experimental temperature vector $\hat{\mathbf{T}}$ is assumed to be related with a constant standard deviation and not correlated, in the domain of validity of the previous asymptotic expansion, then, the optimal estimator of the parameters vector $\left[\beta_{1}, \beta_{2}\right]$ is obtained by :

$$
\left[\begin{array}{l}
\hat{\beta}_{1} \\
\hat{\beta}_{2}
\end{array}\right]=\left(\mathbf{X}^{\mathbf{t}} \mathbf{X}\right)^{-1} \mathbf{X}^{\mathbf{t}} \hat{\mathbf{T}}
$$

The linear approximation allows not only the estimation of the parameters, but also the confidence interval of this estimation to be studied.

The method can be used whatever the length of the vector $\hat{\mathbf{T}}$. These expressions can be implemented simultaneously with all of the pixels of the image. Thus, the matrix product $\mathbf{X}^{\mathbf{t}} \hat{\mathbf{T}}$ can be incremented and consists in a real time weighting. The choice of the weighting is linked to the choice of the estimation strategy (estimation of $L, \rho c$ or $\lambda$ ). The terms of the sensitivity matrix are theoretically calculated with the references values or the averaged images. This sequential method considerably eases the problems of storage and images manipulation. It is very suitable for a simple Non Destructive Evaluation.

Moreover, $f(t)$ can be "measured" on the data, because at each time step, the average of the image is a filtered approximation of $f(t)$. The computation of the logarithmic derivative of $f(t)$ would then give a suitable method applicable without any idea about the knowledge of the nominal values of the thermophysical properties of the considered sample. Unfortunately, the logarithmic derivative of this experimental signal is not easy with simple finite differences methods. In order to overcome such difficulties Shepard et al [9] proposed intuitively a logarithmic time-fitting each pixel signal and Rajic[2] to consider the SVD of the global data cube.

In order to conveniently process the great amount of data provided by the flash NDE experiment, Shepard proposed to decompose the signal with a polynomial fitting, such as:

$$
\operatorname{Ln}(T(x, y, z=0, t))=\beta_{0}(x, y)+\beta_{1}(x, y) \operatorname{Ln}(t)+\beta_{2}(x, y) L n^{2}(t)+\ldots
$$

Such decomposition has no physical meaning because the new parameter vector is not related to a physical model, but the time-logarithmic derivative (considered by Shepard) appears to be very well correlated with the depth or thermophysical properties changes of the tested samples. The calculation of the logarithmic derivative is then taking the advantages related in section 2-2. It is also a very efficient and convenient method in order to reduce and manipulate the great amount of data (only $N$ images corresponding to the degree of the polynomial expressions are to be manipulated). An other way to reduce the amount of data consists in considering the SVD of the information cube. Rajic [2] proposed the SVD decomposition (explained in 2.5) such as:

$$
T(x, y, z=0, t)=\sum_{k=1}^{K} u_{k}(x) \gamma_{k} v_{k}(t)
$$

Such data obtained from NDE experiments appears to be nicely reduced by 2 or 3 terms of the previous serie. Generally, the $\mathbf{U}_{1}$ vector (or $u_{1}(x)$ function) is giving a good approximation of the spatial energy distribution. The $\mathbf{V}_{\mathbf{1}}$ vector (or $v_{1}(t)$

function) is related to $f(t)$. The $\mathbf{U}_{2}$ vector (or $u_{2}(x)$ function) is giving a good approximation of the defects localisation.

Bamford et al [4] proposed to compare the asymptotic expansion firstly explained in this section to the previous SVD decomposition. The slight differences are coming from the non-orthogonality of the functions in the asymptotic case.

In fact all the methods presented in section 3.1 are very similar. They consist in projecting the data cube in a suitable basis of functions (in space or in time, physical or not) and then to try to process the signal with a physical model. Such methods are suitable because every pixel the whole image related to the analysed field are of interest. Such asymptotic approaches are very convenient in the case where the thermophysical properties mapping is slightly varying versus mechanical stresses. The result shown in the figure 7 was used for Ceramic Matrix Composite (CMC) characterization (see [10]). 

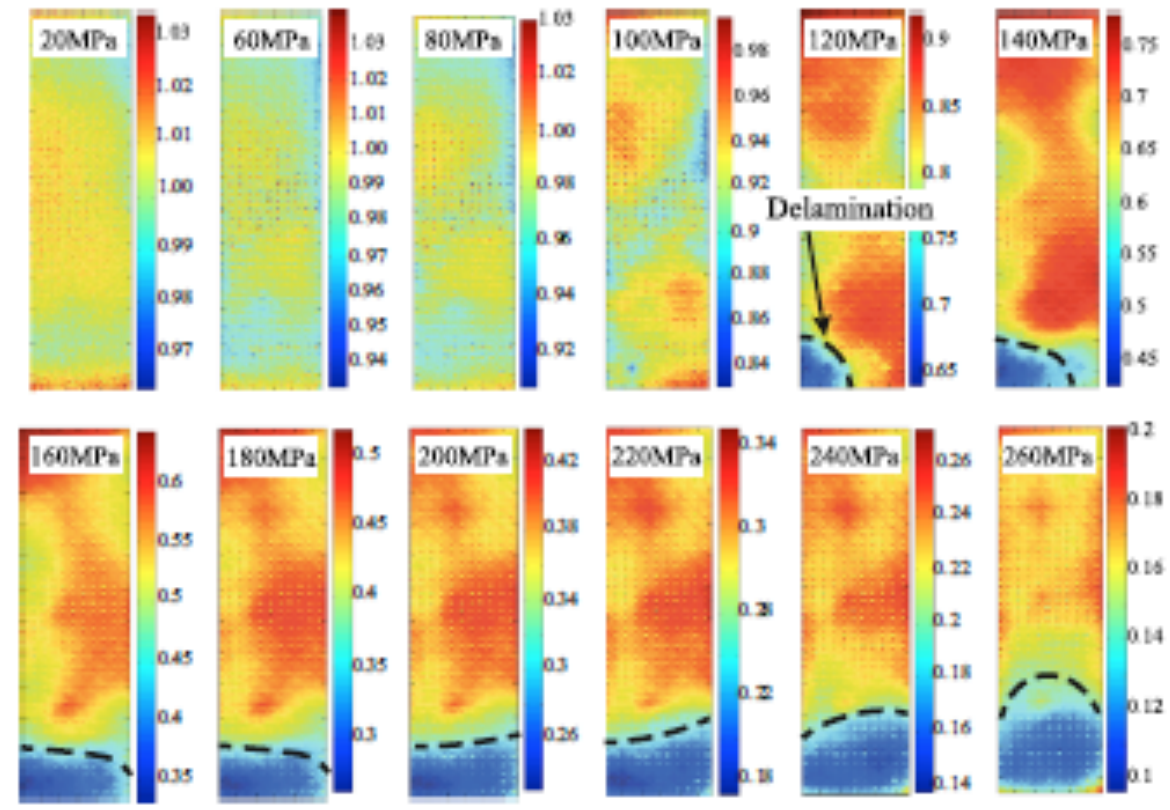

Fig 6: Evolution of the transverse diffusivity mapping of a CMC sample under a tensile test. They illustrate the intiation and the propagation of the delamination (from [10]).

In the next section (application to microfluidic devices), an other strategy is proposed. It consists in using the physical model in order to reduce or eliminate the non useful data (because nothing physically happens or because the sensor is providing a wrong signal).

\subsection{Application to microfluidic devices characterization}

The micro fabrication techniques originally devoted to electronics have reach spectacular advances in more general domains such as chemical micro reactors with micro fluidic channels (lab on chip see [11], [12]). Such systems are very profitable because the low fluid or solid mass requirements and small volumes induce low cost fabrication. It leads new ways to design and intensify the experiments such as screening for catalyst testing related to chemical engineering see [13]). Microdevices also have a lot of interesting characteristics in the field of heat transfer (high speed of the diffusive transfer, possibility of implementing localised heat sources, high surface/volume ratio and intensive local heat flux with low global energy consumption). One of the main drawbacks is related to the instrumentation. Particularly, for temperature measurement, the IR thermography is a non intrusive, way for the recording and processing of a large amount of data. The main challenge is then to set out processing methods of the surface temperature field of such systems through a heat transfer model. Once again, the main characteristics of such data processing is related to temperature differences or derivatives versus time and space. This will be illustrated with the characterization of a simple transient in plane diffusion on a thin plate and then the characterization of convective effect caused by a microchannel sticked to a thin conductive plate (see [14]).

The simple in plane diffusion with source term is met usually in microelectronics. The objective can be to estimate the space and time distribution of energy given on a silicon plate from electronic chips from the observed transient temperature fields. It can also illustrate the case where a single droplet of reactant is deposited on the homogeneous conductive plate (a glass or silicon thin layer). A classical model of the temperature field with simplified assumptions, is given with the following expression:

$$
\frac{\partial T}{\partial t}=a\left(\frac{\partial^{2} T}{\partial x^{2}}+\frac{\partial^{2} T}{\partial y^{2}}\right)+Q(x, y, t) / \rho c
$$

The estimation method of the source term field : $Q(x, y, t)$ in $W^{-3}$ from a sequence of temperature images illustrated by figure 7-a and figure 7-b consists in considering the Laplacian of the temperature field $: \Delta T=\left(\frac{\partial^{2} T}{\partial x^{2}}+\frac{\partial^{2} T}{\partial y^{2}}\right)$ and the time derivative $\partial T / \partial t$ at each time step and each pixel location, with a previous calibration for the knowledge of the thermal diffusivity $a$. 

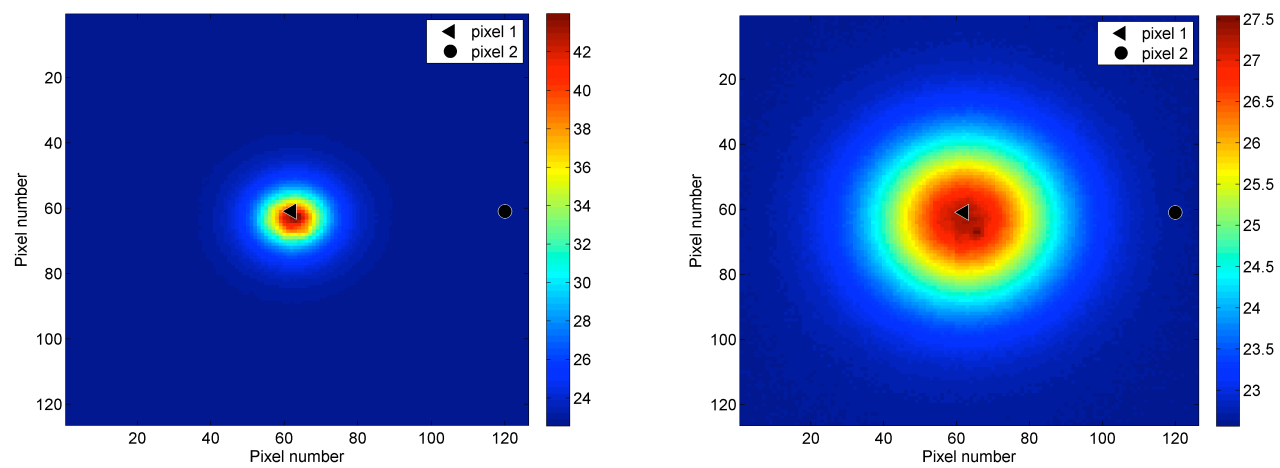

Fig. 7: Temperature response field obtained with a step heating made with a laser spot of 200 micrometers of diameter on a glass plate of $0.2 \mathrm{~mm}$ thick, at two different times. (a-Image obtained at a short time $\quad(t=0.8 \mathrm{~s}$ after the beginning of the heating), bImage obtained at a longer time $(t=1.6 \mathrm{~s})$. The pixel size is $10 \mu \mathrm{m}$.

One illustration of a possible processing is to study the linear relation between Laplacian and time derivative at each pixel of the image (see figure 8). Such derivatives are obtained from finite differences versus space or time of the temperature information related to the pixels regularly spaced versus time and space.

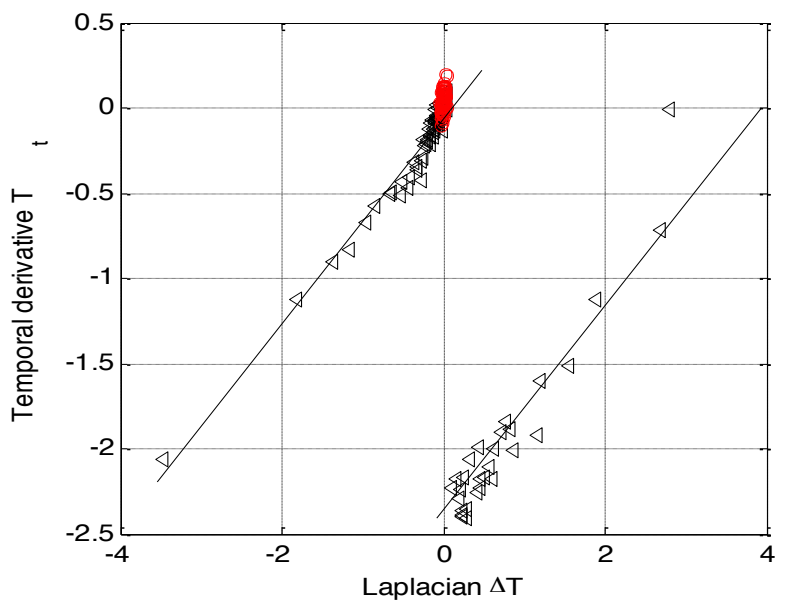

Fig 8: Time derivative versus Laplacian of temperature; a- for an irrelevant pixel out of the heating zone (in red), b-for a pixel inside the heating zone (in black).

On figure 8 , the straight line with origin ordinate is corresponding to pixel where a constant heating occurs. The straight line passing through the origin is corresponding to a case of pure diffusion (when the temperature field is relaxing after the heating). The source term can then be estimated versus space independently of the transport property (the thermal diffusivity: a ). A criterion suitable to detect such zones is to consider the local correlation between the laplacian $\Delta T$ ' ${ }^{\prime}$ and the time derivative here noted as $\partial T=\partial T / \partial t$ :

$$
\rho_{i, j}^{F_{t}}=\frac{\sum_{F_{t}} \Delta T_{i, j}^{k} \partial T_{i, j}^{k}}{{\sqrt{\sum_{F_{t}} \Delta T_{i, j}^{k}}}^{2} \sqrt{\sum_{F_{t}} \partial T_{i, j}^{k}}}
$$

where $F_{t}$ is a temporal window such as: $F_{t}=[k, k+I t]$ with $k \in[1, N-I t], k$ is the time step number and $I t$ the width of the time window. If such a coefficient is near from 1 , a diffusivity parameter is then estimable. If the correlation coefficient is near from zero the pixel or the area is irrelevant and must be discarded.

This example is demonstrating that the great amount of thermographic data coupled with computer facilities is offering large possibilities to estimate not only one source term but also a regularly spaced distribution of sources. It offers then the possibility to design thousands of simultaneous independent experiments. It is then also possible to extend the experimental configurations to microchannels.

The idea which consists in implementing a slow in-plane transfer in a conductive plate is here used to estimate the convective transport effect due to a flow in a micro channel (of diameter from 10 to 100 miccrometers) stuck to a thermally conductive plate (see figure 9). The technical details of the experiment are described in [15]. 

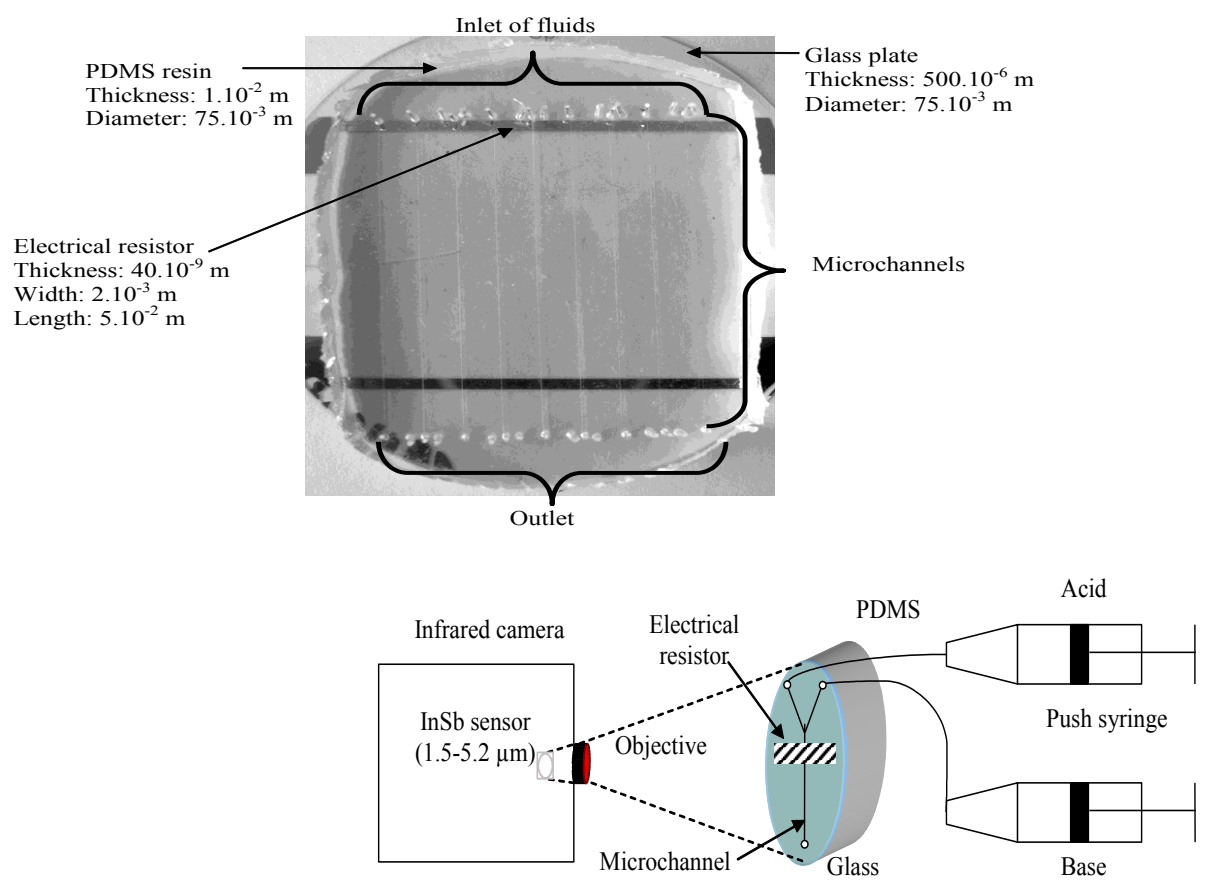

Fig 9. Microfluidic chip, and principles of the microreactor experiment

The first step of the study will here consist in comparing and estimating the convective effect in the channel to the $2 \mathrm{D}$ diffusion inside the conductive front plate.

Here, the local laplacian of the temperature field is compared to the local temperature gradient versus the direction of the channel ( $x$-direction) in order to estimate a Peclet Field. In order to realize a calibration experiment a thin heating resistor is deposited at the entrance of the channel on the conductive plate. An in-plane reference permanent temperature field $T_{i, j}^{0}$ is obtained (see figure 11-a) with stationary pure water inside the channel and the heating on the left of the plate.

Then, a perturbed temperature field $T_{i, j}$ is obtained with a water flow inside the channel (see Figure 10-b). The deformation of the isothermal lines is clearly shown by comparison between figure 10-a and 10-b.
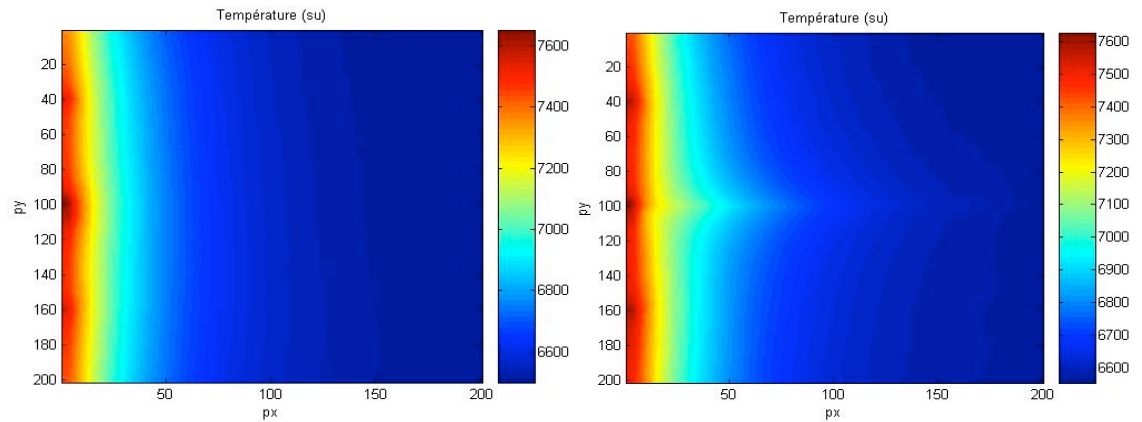

Fig 10: a-temperature field $T_{i, j}^{0}$ (without flow); b-temperature field $T_{i, j}$ (with micro flow versus the $x$-direction)

In order to estimate the non dimensional velocity field compared to diffusion, a Peclet field $P e_{i, j}$ is locally defined such as:

$$
P e_{i, j}=\Delta\left(T_{i, j}-T_{i, j}^{0}\right) /\left(\partial\left(T_{i, j}\right) / \partial x\right)
$$

It is very important to remark that such estimation (see figure 12) is giving a constant non zero velocity well estimated inside the channel location and with a poor noise only in the sharp temperature gradient zone. A more detailed analysis with a correlation analysis is given in [16]. 


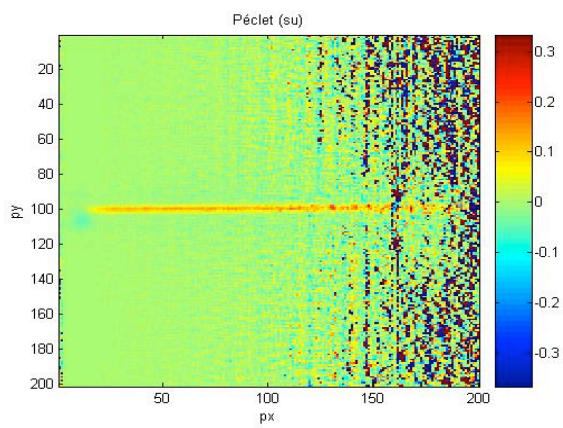

Fig 11 : Peclet field estimation $P e_{i, j}$ from figures 10.

Such Peclet field can then be used in order to estimate the source term along the channel in the case of a specific microreactions in very near conditions of flow rate and fluids. For example, both liquids $(\mathrm{HCl}$ and $\mathrm{NaOH}$, concentration of 0.25 $\mathrm{mol} / \mathrm{l})$ are assumed to be with the same thermophysical properties as pure water. Then, the measurements were realised at the same flow rate as the previous experiment and the infrared camera allows the detection of the chemical heat flux created by the reaction (see [15]).

From these first considerations, the microfluidic applications have been largely improved. It is now possible to estimate the enthalpy of chemical reactions in a lot of channel configurations and micro or millifluidic devices (see [14] to [18]). A special attention was paid to droplet flows and the related analysis of the heat release of such complex systems (see [19]).

More recently, a complementary step was devoted to the detection of the transmission of wide-band multispectral infrared sources in such chemical devices. There is now a challenge to combine heat transfer and chemical analysis is order to estimate simultaneously thermophysical and thermochemical parameters related to coupled heat and mass transport in such devices.

\subsection{Applications to thermal multispectral tomography}

The transmission of wide-band multispectral infrared sources through heterogeneous objects can be detected by simple thin opaque or semitransparent thin screens. Such screens are then acting as bolometers where the transient temperature field can be analysed by a unique IR thermography device. Our team is developing at this moment a large range of instruments based on terahertz radiation (or long wave infrared radiation (see [20]) or wide band infrared multispectral radiation, coupled with thermal imaging systems (see [21]). One of the key obstacle is to detect the thermal effect of very low-energy sources absorbed in a short time by a thin screen.

One illustration of this estimation problem is corresponding to the $1 \mathrm{D}$ observation of the transient temperature response $T(x, t)$ to a pulse heating of a laser spot on a beam graduated following the $x$-direction. Thanks to infrared thermography, the instantaneous temperature fields can be measured by infrared thermography at $N_{x}$ different space steps $x_{i}$ (lying between $O$ and $L$ ) and at $N_{t}$ different time steps $t_{j}$ at $N_{t}$ different time steps (lying between 0 and $t_{m a x}$ ). It is classically assumed that the measurement errors at each space and time step are uncorrelated, of zero mean and on uniform variance $\sigma^{2}$ or standard deviation $\sigma$. The processing of the observable temperature response: $\hat{T}(x, t)$ will be illustrated in these examples. The objective, is to use the great amount of data $\left(N_{x} \times N_{t}\right.$ data) in order to filter the estimation of the initial temperature field $T(x, t=0)$. The general 1-D problem of diffusion with initial condition, without internal source, in an infinite medium can be presented under the following system

$$
\frac{\partial T}{\partial t}=a \frac{\partial^{2} T}{\partial x^{2}}
$$

with at initial time : $\quad T(x, t=0)=f(x)$

It is here assumed that the 1D medium is infinite and the temperature is zero for $T(x, t)$ and $f(x)$ when $x$ tend to infinite.

A direct solution of this system is obtained by implementing a space Fourier transform (see [22], [23]). Once again the Fourier transform is a suitable way to consider the Laplacian or second derivative of the temperature versus space:

$$
\theta\left(\alpha_{n}, t\right)=\int_{0}^{L} T(x, t) \cos \left(\alpha_{n} x\right) d x \quad \text { with } \quad \alpha_{n}=n \pi / L ; n>0 r=0
$$

$L$ is here assumed as a "large" bound of the $x$-domain, where the temperature is zero and no gradient is to be considered. The previous system becomes an ordinary differential equation, after Fourier transform:

$$
-\alpha_{n}^{2} \theta\left(\alpha_{n}, t\right)=\frac{1}{a} \frac{d \theta}{d t}
$$


The initial condition becomes:

$$
\theta\left(\alpha_{n}, 0\right)=F\left(\alpha_{n}\right)
$$

The solution of the forward problem is then:

$$
\theta\left(\alpha_{n}, t\right)=\theta\left(\alpha_{n}, 0\right) \exp \left(-a \alpha_{n}^{2} t\right) \quad \text { for every } n
$$

Or in the real space:

$$
T(x, t)=\frac{1}{L}\left[\theta(0,0)+\frac{1}{2} \sum_{n=1}^{\infty} \cos \left(\alpha_{n} x\right) \theta\left(\alpha_{n}, 0\right) \exp \left(-a \alpha_{n}^{2} t\right)\right]
$$

Such an estimator is decreasing the influence of the measurement noise on the initial estimator: $\hat{\theta}\left(\alpha_{n}, t_{j}\right)$ the terms of the covariance matrix of the previous estimator are then:

$$
\operatorname{cov}(\hat{\hat{\theta}}(0,0))=\frac{\sigma^{2} L^{2}}{N_{x} N_{t}} \text { and } \operatorname{cov}\left(\hat{\hat{\theta}}\left(\alpha_{n}, 0\right)\right)=\frac{\sigma^{2} L^{2}}{N_{x}}\left(\frac{1}{\sum_{j=0}^{N_{t}} \exp \left(-2 a \alpha_{n}^{2} t_{j}\right)}\right) \text { if } n \text { non zero. }
$$

Then, the standard deviation of the estimator in the $x$-space $\left(\sigma_{E}\right)$ are in the following interval:

$$
\sigma / \sqrt{N_{t}}<\sigma_{E}<\sigma
$$

It is necessary to remark that the processing of such amount of data is efficient if the time steps related to the observation are near from 0 . It becomes then a convenient way to avoid the blurring effects due to diffusion. Unfortunately, the key parameter is here the perfect knowledge of the thermal diffusivity: $a$. Very often such parameter is unknown and is not suitably measurable directly on the instrumental device. Once again a Singular Value Decomposition is a quite similar decomposition as Fourier, but does not require the knowledge of a physical model and the of the diffusivity parameter.

An extension of theses remarks to a $2 \mathrm{D}$ separable case are then even more efficient. If the initial condition is then depending on two space variables: $x$ and $y$ and that such initial condition is separable such as:

$$
f(x, y)=f_{x}(x) f_{y}(y)
$$

With the Fourier transform method, the solution of the 2D problem is then a product of two series depending on $x$ and $t$ and $y$ and $t$, such as :

$$
T(x, y, t)=\frac{1}{L}\left[\theta(0,0,0)+\frac{1}{2} \sum_{n=1}^{\infty} \cos \left(\alpha_{n} x\right) \theta\left(\alpha_{n}, 0,0\right) \exp \left(-a \alpha_{n}^{2} t\right)\right] \frac{1}{l}\left[\theta(0,0,0)+\frac{1}{2} \sum_{m=1}^{\infty} \cos \left(\beta_{m} y\right) \theta\left(0, \beta_{m}, 0\right) \exp \left(-a \beta_{m}^{2} t\right)\right]
$$

$\beta_{m}$ is a spatial frequency related to the $y$-direction, equivalent to $\alpha_{n}$ related to the $x$-direction.

The analogous estimator in the $2 \mathrm{D}$ Fourier space is also separable and gives:

$$
\hat{\hat{\theta}}\left(\alpha_{n}, \beta_{m}, 0\right)=\frac{\sum_{j=0}^{N_{t}} \hat{\theta}\left(\alpha_{n}, 0, t_{j}\right) \exp \left(-a \alpha_{n}^{2} t_{j}\right)}{\sum_{j=0}^{N_{t}} \exp \left(-2 a \alpha_{n}^{2} t_{j}\right)} \frac{\sum_{j=0}^{N_{t}} \hat{\theta}\left(0, \beta_{m}, t_{j}\right) \exp \left(-a \beta_{m}^{2} t_{j}\right)}{\sum_{j=0}^{N_{t}} \exp \left(-2 a \beta_{m}^{2} t_{j}\right)}
$$

In such a particular case where each instantaneous temperature field is separable versus the $x$-direction and $y$-direction, it is suitable to consider preliminarily a SVD, versus $x$ and $y$ at each time step such as:

$$
\hat{T}\left(x_{i}, y_{j}, t\right)=\sum_{k} U_{k}\left(x_{i}, t\right) W_{k}(t) V_{k}\left(y_{j}, t\right)
$$

Only the first term of such a decomposition is then to be taken into account. The order of magnitude of the second term must be much lower than the first one. It is then suitable to consider a secondary decomposition versus the $x$ and $y$ directions such as:

$$
U_{1}\left(x_{i}, t_{j}\right)=\sum_{r=1}^{r 0 x} U_{r}^{x}\left(x_{i}\right) W_{k}^{x} V_{r}^{x}\left(t_{j}\right) \quad \text { and } \quad V_{1}\left(y_{i}, t_{j}\right)=\sum_{r=1}^{r 0 y} U_{r}^{y}\left(y_{i}\right) W_{k}^{y} V_{r}^{y}\left(t_{j}\right)
$$


The advantage of such a truncated double-decomposition is to operate a strong data reduction. For example, if $N_{x}=N_{y}=N_{t}=1000$; the global number of data to be processed is $10^{9}$. But the successive truncations allow to reduce the number of data to be considered to 12000 (if $r 0 x=r 0 y=3$ ). The measurement noise attenuation is than more than 100 times higher (see [22]). The figures 12-a to 12-c are illustrating the 2D temperature response to a punctual heat pulse lost in the noise. It shows that even when the noise level is of the same order of magnitude as the signal, the SVD decomposition allows the detection of a very localised point source. This argument is in favour of the bolometric detection of localised radiative source of very low amplitude such as terahertz radiations (see [20]), or in the case of multispectral imaging where the low-energy of each radiative band must be considered (see [21]). Other attempts are studied is order to gather all the previous remarks in the case of Gaussian temperature distributions (threshold by correlation, separation by SVD, space polynomial fitting, TSR ...), in the paper presented by Gaverina et al [24] at the QIRT Asia Conference.
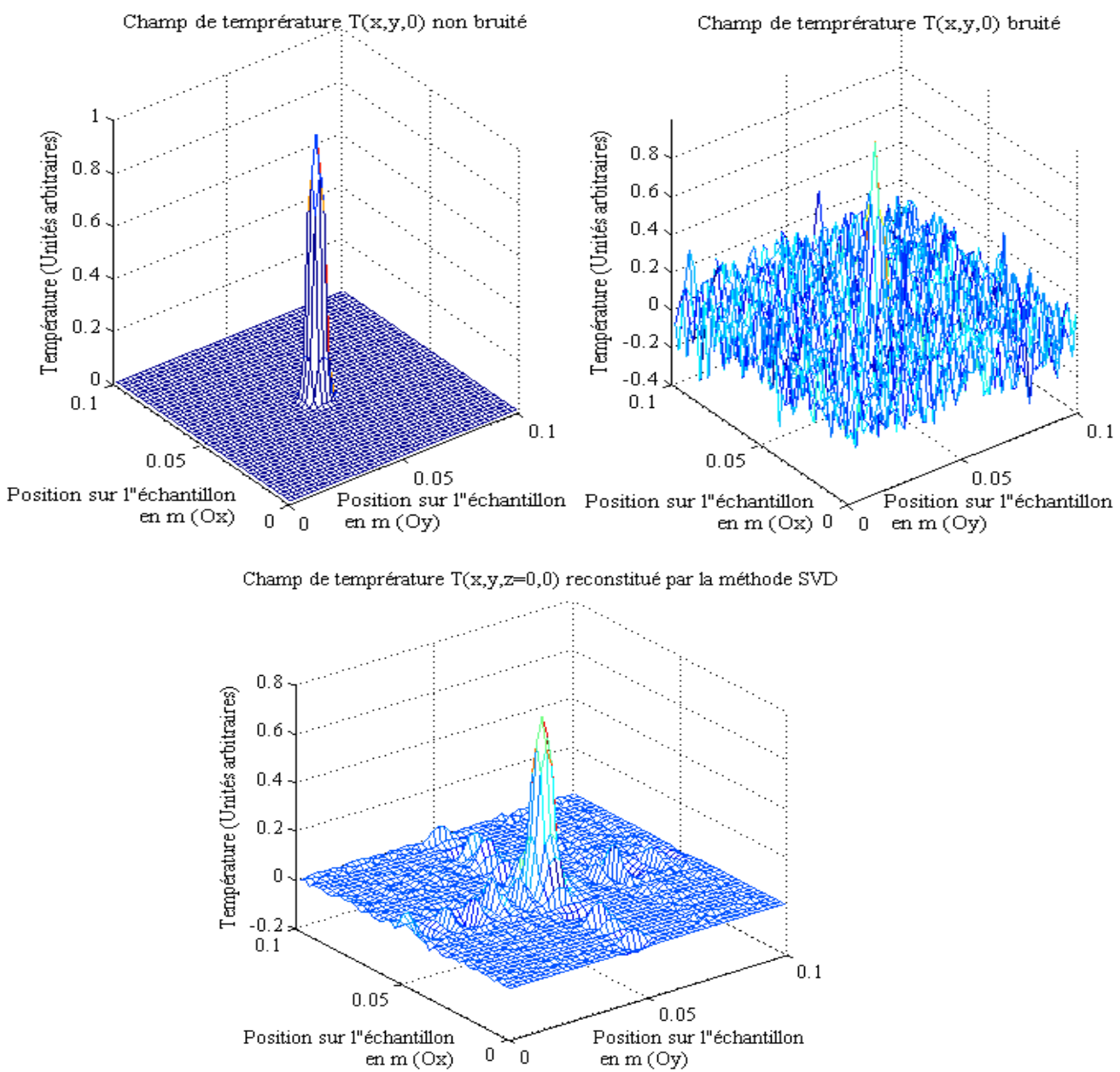

Fig. 12 : Initial temperature field (expression (60) a-without noise, b-with a high noise/signal ratio, c- filtered and reduced by SVD with $r_{0}=3$.

\section{Conclusion}

The time-space thermal signal is offering a great amount of data which must be processed from several points of views. Several strategies are available in order to process the signal in relation with a physical model. Often it will consist in projecting the signal in a function basis which can come from intuition, statistical processing or physical analysis. This projection is advantageous in order to filter the random noise, to reduce the great amount of data and to conveniently manipulate and estimate the parameters. But the projection is not always the most suitable strategy. The direct consideration of a physical model will allow to eliminate or discriminate the data correlated or not with a chosen physical phenomenon (one example has been evocated with the estimation of a thermal diffusivity field).

Such considerations was also a common thread in order to present the IR thermography activities of the I2M at Bordeaux University (France).

The perspective of these works is now clearly devoted to the exploration of multispectral instrumentation with IR cameras. Especially, the spectral derivative of temperature or luminance fields will be a new direction for the signal processing of an amount of data always bigger with 4 dimensions to be considered. 


\section{REFERENCES}

[1] Thikonov A. and Arsenine V., 1977 Solutions for ill posed problems, Washington: Winston \& So

[2] Rajic N., Principal component thermography for flaw contrast enhancement and flaw depth characterisation in composite structures, Composite Structures, Volume 58, Issue 4, December 2002, Pages 521-528

[3] Bamford M., Batsale J.C., Fudym O., Nodal and Modal Strategies for longitudinal Thermal Diffusivity Profile Estimation. Application to the non-destructive Evaluation of $\mathrm{SiC} / \mathrm{SiC}$ composites under uniaxial tensile tests, Infrared Physics and technology (2008), Volume 52, Issue 1, January 2009, Pages 1-13

[4] Bamford M., Batsale J.C.: Analytical singular value decomposition of infrared image sequences : microcrack detection on ceramic composites under mechanical stresses. Comptes Rendus Ac. Sci. - Mecanique, 336(5):440-447, 2008.

[5] Bamford M., Florian M., Vignoles G.L., Batsale J.-C., Cairo C.A.A. ; Maillé L.: Global and local characterization of the thermal diffusivities of sicf/sic composites with infrared thermography and flash method. Composites Science and Technology, 69(7-8):1131-1141, 2009.

[6] Balageas D., Delpech P., Boscher D., Deom A., "New developments in stimulated infrared thermography applied to non destructive evaluation of laminates", Review on Progress in Quantitative Non-Destructive Testing, ED Thompson and Chimienti (Plenum Press, New York, 1991, Vol 10 A, pp 1073-1081

[7] Parker WJ, Jenkins, W., Abott J.., 1961 "Flash method of determining thermal diffusivity, Heat capacity and thermal conductivity", Journal Appl. Phys., vol 32, No 9, pp 1679-1684,

[8] Mourand D., Batsale J.C., Sequential method for thermal diffusivities discrimination by infrared thermography, High Temperatures - High Pressures, vol 33 (2001) 127-134

[9] S.M. Shepard, Y.L. Hou, T. Ahmed and J.R. Lhota, "Reference-free Interpretation of FlashThermography Data", Insight, Volume 48, No. 5, British Institute of NDT, May 2006, pp.298-307.

[10] EL Yagoubi j., Lamon J. Batsale JC, Dhote J., Le Flem M., Multiscale Thermal charactérization of Méchanically loaded Ceramic Matric Composites, Estimation of the Diffusivity at Mico/Méso Scale, Experimental Mechanics DOI 10.1007/s11340-014-9976-x.

[11] K.F. Jensen, Microreaction engineering-is small better ?, Chemical Engineering Science, 2001, pp $293-303$.

[12] K.P. Möllmann, N. Lutz and M. Vollmer, Thermography of microsystems, Inframation proceedings, ITC 104 A, $07-$ $27,2004$.

[13] S. Senkan, K. Krantz, S. Ozturk, V. Zengin, I. Onal, High-throughput Testing of Heterogeneous Catalyst Libraries Using Array Microreactors and Mass Spectrometry, Angewandte Chemie- International Edition, 38, 27942799, 1999

[14] Pradere C., Morikawa J., Toutain J., Batsale J.-C., Hayakawa E., Hashimoto T., Microscale thermography of freezing biological cells in view of cryopreservation, Quantitative IR Thermography Journal 6 (2009) 37-61.

[15] Pradere C., M. Joanicot, J.C. Batsale, J. Toutain, C. Gourdon, Processing of temperature field in chemical microreactors with infrared thermography, QIRT Journal, vol 3 n1/2006, pp117-135

[16] Ravey C., Pradere C., Regnier N., Batsale J.C., New temperature field processing from IR camera for thermal diffusivity and calorimetric non-intrusive measurements in microfluidics systems. QIRT 9, pp. 79-98, DOI 10.1080/17686733.2012.682878, 2012.

[17] Hany C., Pradere J. Toutain J., Batsale JC., Joanicot M., A millifluidic calorimeter without contact for reaction enthalpy and kinetics measurements, QIRT Conference Krakow 2008.

[18] C. Hany, C. Pradere, J. Toutain, J.-C. Batsale, A millifluidic calorime-ter with infrared thermography for the measurement of chemical reactionenthalpy and kinetics, QIRT Journal 5 (2008) 211-219.

[19] M. Romano, C. Pradere, F. Sarrazin, J. Toutain, J.C. Batsale ; Enthalpy, kinetics and mixing characterization in droplet-flow millifluidic device by infrared thermography; Chemical Engineering Journal (2015), http://dx.doi.org/10.1016/j.cej.2015.03.071

[20] Pradere C. Caumes J.P., Salort S., Balageas D., Chassagne B., Batsale JC.,, Photothermal converters for quantitative 2D and 3D real-time terahertz imaging. Qunatitative infrared Thermography, pp 217-235, Vol 7 issue 2, 2010.

[21] Romano M., Ndiaye C., Duphil A., Sommier A., Morikawa J., Mascetti J., Batsale J.C., Servant L., Pradere C., Fast infrared imaging spectroscopy technique, (FIIST), Infrared Physics and Technology, 68 (2015), 152-158

[22] Avayzyan V., Etude de champs de temperature séparables avec une double decomposition en valeurs singulières Quelques applications à la caracterisation de proprieties thermophysiques de matériaux et au contrôle Non-Destructif, Doctorate Thesis, Université de Bordeaux, France 2012

[23] D. Maillet, S. André, J.C. Batsale, A. Degiovanni, C. Moyne, Thermal Quadrupoles - Solving the Heat Equation through Integral transforms, Editeur : John Wiley \& Sons, Ltd, Chichester, 370 pages, October 2000.

[24] Gaverina L., Batsale JC., Pradere C. , Mourand D., Several considerations about a pulsed flying spot method implemented with IR thermography, first QIRT Asia Conference, July 2015. 